\title{
Advantages of ddPCR in detection of PLP1 duplications
}

\author{
Taichi Imaizumi $^{1,2}$, Keiko Yamamoto-Shimojima ${ }^{2,3}$, Toshiyuki Yamamoto ${ }^{2,3, *}$ \\ ${ }^{1}$ Department of Pediatrics, St. Marianna University School of medicine, Kawasaki, Japan; \\ ${ }^{2}$ Institute of Medical Genetics, Tokyo Women's Medical University, Tokyo, Japan; \\ ${ }^{3}$ Tokyo Women's Medical University Institute of Integrated Medical Sciences, Tokyo, Japan.
}

\begin{abstract}
Summary Pelizaeus-Merzbacher disease (PMD) is an X-linked, recessively inherited disorder associated with hypomyelination in the brain white matter. Mutations involving the proteolipid protein 1 gene (PLP1) located on Xq22.2 are responsible for PMD. PLP1 duplication is the major genetic abnormality in PMD patients. In this study, we utilized droplet-digital polymerase chain reaction (ddPCR) as a potential method to detect PLP1 duplications. Samples from four PMD patients and one of their mothers were used as positive controls. They had been previously diagnosed as having an additional PLP1 copy by chromosomal microarray testing. Genomic copy number of $P L P 1$ was analyzed in triplicate experiments and compared with reference genes XIST and $A R$ on the X-chromosome, and RPP30 and RPPHI on the autosomes. As a result, precise results were obtained for each triplicate procedure. Thus, we concluded that triplicate experiments are no longer necessary. Compared to other methods, including fluorescence in-situ hybridization, multiplex ligation-dependent probe amplification, chromosomal microarray testing, and quantitative PCR, we were able to establish ddPCR results rapidly with very small amounts of DNA. In conclusion, we showed that ddPCR can be a potential diagnostic tool to confirm genomic copy number as a routine clinical application, including in prenatal diagnostic settings.
\end{abstract}

Keywords: PLP1 duplications, droplet digital polymerase chain reaction, copy number variations

\section{Introduction}

Pelizaeus-Merzbacher disease (PMD) is a genetic disorder associated with hypomyelination in brain white matter, and most patients with PMD exhibit motor developmental delay, hypotonia, horizontal nystagmus, and progressive spasticity (1). The proteolipid protein 1 gene $(P L P 1)$ located on Xq22.2 is responsible for PMD. Thus, PMD is recognized as an $\mathrm{X}$-linked recessive disorder and most patients are male. Their PLP1 abnormalities are often inherited from their carrier mothers. It is also known that twothirds of PMD patients generally show chromosomal microduplications involving the Xq22.2 region, which

Released online in J-STAGE as advance publication August 20, 2019.

*Address correspondence to:

Dr. Toshiyuki Yamamoto, Institute of Medical Genetics, Tokyo Women's Medical University, 8-1 Kawada-cho, Shinjuku-ward, Tokyo 162-8666, Japan.

E-mail: yamamoto.toshiyuki@twmu.ac.jp harbors $P L P 1$ (2). For these reasons, it is recommended to first screen for $P L P 1$ duplication in cases of patients suspected of having PMD. In such cases, fluorescence in-situ hybridization (FISH) and multiplex ligationdependent probe amplification (MLPA) methods have been used previously. The more efficient method is chromosomal microarray testing, because this method can detect not only duplications but also identify extent of duplications.

When we detected PLPI duplications in probands, diagnosis of carrier status of their mothers is often required. Furthermore, when mothers carry PLP1 duplications, prenatal diagnosis is often required for subsequent pregnancies. In such cases, analytical methods need to fulfill certain conditions. Results should be rapidly and precisely obtained. It would be advantageous if the amounts of DNA required for these tests are small. Consequently, chromosomal microarray testing has limitations for the purpose of this screening.

The aim of this study was to establish a simple and rapid detection method to confirm PLP1 duplications. With this aim, we utilized droplet-digital polymerase 
chain reaction (ddPCR) as a potential method as a 'proof-of-concept'.

\section{Materials and Methods}

\subsection{Materials}

Positive control samples were prepared as described in previous studies (3). Four PMD patients and one carrier mother were used. All patients had already been diagnosed to have PLP1 duplications by chromosomal microarray testing. The sizes of the duplicated regions are shown in Table 1. Control samples from 1 normal male and 1 normal female were also used.

This study was performed in accordance with the Declaration of Helsinki and approved by the ethics committee of the Tokyo Women's Medical University. Written informed consent was obtained from patients and their parents before peripheral blood samples were acquired.

\subsection{Methods}

By use of the QIAamp DNA extraction kit (QIAGEN, Hilden, Germany), genomic DNA was extracted from peripheral blood samples. DNA concentration was calculated using a Qubit ${ }^{\circledR} 2.0$ Fluorometer (Life Technologies, Carlsbad, CA, USA). PLP1 was the target in this study. X inactive specific transcript (XIST), androgen receptor $(A R)$, ribonuclease $\mathrm{P} / \mathrm{MRP} 30 \mathrm{kDa}$ subunit $(R P P 30)$ and ribonuclease P RNA component $\mathrm{H} 1(R P P H 1)$ genes were selected as references. XIST (Xq13) and $A R$ (Xq12), were used as references for the X-chromosome. RPP30 (10q23) and RPPH1 (14q11) were used as reference for the autosomal chromosomes, as they are located in conserved regions, known to have low frequency of copy number variants $(\mathrm{CNVs})(4,5)$.

Our priority in this study was to confirm the simplicity and accuracy of ddPCR. Because we used an intercalation system rather than $\operatorname{TaqMan}^{\circledR}$ hydrolysis probes, primers were not labeled with fluorescent dyes. We designed the primers with Primer3Plus (https:// primer3plus.com/) and checked for homology using the UCSC Genomic Browser BLAT (https://genome.ucsc. edu/cgi-bin/hgBlat). Primers details are given in Table 2.

The ddPCR reaction mixtures were prepared as 20 $\mu \mathrm{L}$ total volumes, which included $10 \mu \mathrm{L} 2 \times \mathrm{QX} 200$ ddPCR EvaGreen Supermix (Bio-Rad Laboratories, Hercules, CA), $1 \mu \mathrm{L}$ each of the forward and reverse primers $(1 \mu \mathrm{M}), 1 \mu \mathrm{L}$ DNA $(20 \mathrm{ng} / \mu \mathrm{L})$, and $7 \mu \mathrm{L}$ RNase/DNase-free water. We loaded $20 \mu \mathrm{L}$ of reaction mixture and $70 \mu \mathrm{L}$ of QX200 Droplet Generation Oil for EvaGreen (BioRad Laboratories) into a QX100/200 DG cartridge (Bio-Rad Laboratories), which was transferred into a QX200 Droplet Generator (Bio-Rad Laboratories). Droplets containing $40 \mu \mathrm{L}$ of oil and sample emulsion were transferred into clean 96-well
Table 1. Summary of the samples

\begin{tabular}{ll}
\hline Samples & Duplication sizes \\
\hline Patient 1 & $648-\mathrm{Kb}$ \\
Patient 2 & $603-\mathrm{kb}$ \\
Patient 3 & $656-\mathrm{kb}$ \\
Patient 4 & $948-\mathrm{kb}$ \\
Carrier mother & $603-\mathrm{kb}$ \\
Control male & None \\
Control female & None \\
\hline
\end{tabular}

PCR plates. To avoid contamination and evaporation, the plates were sealed using a PX1 PCR plate sealer (Bio-Rad Laboratories) with pierceable foil heat seals (Bio-Rad Laboratories). Then, PCR was performed with a Gene Amp PCR system 9700 (Thermo Fisher Scientific, Waltham, MA, USA). The thermal cycling conditions were as follows: $95^{\circ} \mathrm{C}$ for $5 \mathrm{~min}(1 \mathrm{cycle})$; then 40 cycles of $95^{\circ} \mathrm{C}$ for $30 \mathrm{~s}$ and $60^{\circ} \mathrm{C}$ for $1 \mathrm{~min} ; 4$ ${ }^{\circ} \mathrm{C}$ for $5 \mathrm{~min}, 90^{\circ} \mathrm{C}$ for $5 \mathrm{~min}$, and then held indefinitely at $4^{\circ} \mathrm{C}$. The ramp rate was $2^{\circ} \mathrm{C} / \mathrm{sec}$ in all steps. After thermal cycling, droplets were analyzed for positive and negative signals using the QX200 droplet reader (Bio-Rad Laboratories). For each ddPCR sample, the same process was performed in triplicate. Data analysis was performed when the number of droplets produced was more than 10,000 .

For data analysis, QuantaSoft Version1.7.4 software (Bio-Rad Laboratories) was used to statistically analyze the obtained data. Genomic copy number was calculated using the reference genes as follows: genomic copy number $=(\mathrm{A} / \mathrm{B}) \times \mathrm{C}$, where $\mathrm{A}$ is the concentration of the target DNA, B is the concentration of the reference DNA, and $\mathrm{C}$ is the number of copies of the reference gene. Because RPPH1 and RPP30 are located on the autosomal chromosomes, both are defined to have two copies. Copy numbers of AR, XIST and PLPI on the X-chromosome were different between males and females. Normal males and females are defined to have one and two copies, respectively.

\section{Results and Discussion}

Copy numbers of $A R, X I S T$, and $P L P 1$ were calculated with two reference genes, RPP3O and RPPH1, and obtained results were summarized in Table 3. Copy numbers of RPP30 and RPPH1 were calculated for each other as a reference. As shown, all replicates showed the copy numbers of RPP3O and RPPH1 close to "2", because these two genes are located on the autosomal chromosomes. In comparison, copy numbers of $A R$ and $X I S T$ were different between males and females. Males and females showed the copy numbers of them as "1" and "2", respectively. It is reasonable because $A R$ and $X I S T$ are located on the X-chromosome. From these findings, it was confirmed that ddPCR system can be used for detection of genomic copy number accurately.

In this study, $P L P 1$ duplications were targeted. Final 
Table 2. Designs of the used primers

\begin{tabular}{lllr}
\hline Targeted genes & Sense primers & Antisense primers & Product length (bp) \\
\hline PLP1 & 5'-TCACAACCCCAAAGCAGCACATTTC-3' & 5'-CGGCTAATTCAAAATCCAGCAAAGGG-3' \\
IRAK1 & 5'-AGCTCTGCATCATCGTCGT-3' & 5'-CCAGCTTCTGGACCATCTTC-3' \\
XIST & 5'-TGAGACCTGAGGACTGCAAA-3' & 5'-AGCTTGGCCAGATTCTCAAA-3' \\
AR & 5'-CCAGCAGAAATGATTGCACTA-3' & 5'-CATTTCCGAAGACGACAAGA-3' \\
RPP30 & 5'-GATTTGGACCTGCGAGCG-3' & 5'-GCGGCTGTCTCCACAAGT-3' \\
RPPH1 & 5'-GTCAGACTGGGCAGGAGATG-3' & 5'-TGGCCGTGAGTCTGTTCC-3' \\
\hline
\end{tabular}

Table 3. Results of ddPCR

\begin{tabular}{|c|c|c|c|c|c|c|c|c|c|}
\hline \multirow{2}{*}{ Samples } & \multicolumn{5}{|c|}{ Versus RPPH1 } & \multicolumn{4}{|c|}{ Versus RPP30 } \\
\hline & & $A R$ & $X I S T$ & RPP30 & PLP1 & $A R$ & $X I S T$ & RPP30 & PLP1 \\
\hline \multirow[t]{3}{*}{ Patient 1} & 1 & 1.08 & 1.10 & 2.14 & 1.84 & 1.00 & 1.04 & 1.86 & 1.72 \\
\hline & 2 & 1.10 & 1.06 & 2.18 & 1.92 & 1.00 & 0.96 & 1.84 & 1.76 \\
\hline & 3 & 1.04 & 1.02 & 2.08 & 1.82 & 1.00 & 0.98 & 1.72 & 1.76 \\
\hline \multicolumn{2}{|l|}{ Average } & 1.07 & 1.06 & 2.13 & 1.86 & 1.00 & 0.99 & 1.81 & 1.75 \\
\hline \multicolumn{2}{|l|}{$\mathrm{SD}$} & 0.02 & 0.02 & 0.03 & 0.03 & 0.00 & 0.02 & 0.04 & 0.01 \\
\hline \multirow[t]{3}{*}{ Patient 2} & 1 & 1.22 & 1.02 & 2.20 & 2.04 & 1.10 & 0.94 & 1.82 & 1.86 \\
\hline & 2 & 1.16 & 1.00 & 2.18 & 2.02 & 1.04 & 0.98 & 1.80 & 1.80 \\
\hline & 3 & 1.20 & 1.14 & 2.32 & 2.10 & 1.02 & 0.98 & 1.72 & 1.88 \\
\hline \multicolumn{2}{|l|}{ Average } & 1.19 & 1.05 & 2.23 & 2.05 & 1.05 & 0.97 & 1.78 & 1.85 \\
\hline \multicolumn{2}{|l|}{ SD } & 0.02 & 0.04 & 0.04 & 0.02 & 0.02 & 0.01 & 0.03 & 0.02 \\
\hline \multirow[t]{3}{*}{ Patient 3} & 1 & 1.02 & 0.98 & 2.02 & 1.76 & 1.02 & 0.98 & 1.98 & 1.74 \\
\hline & 2 & 1.06 & 1.08 & 2.16 & 2.02 & 1.00 & 1.00 & 1.86 & 1.88 \\
\hline & 3 & 1.14 & 1.02 & 2.14 & 1.98 & 1.06 & 0.96 & 1.86 & 1.84 \\
\hline \multicolumn{2}{|l|}{ Average } & 1.07 & 1.03 & 2.11 & 1.92 & 1.03 & 0.98 & 1.90 & 1.82 \\
\hline \multicolumn{2}{|l|}{ SD } & 0.04 & 0.03 & 0.04 & 0.08 & 0.02 & 0.01 & 0.04 & 0.04 \\
\hline \multirow[t]{3}{*}{ Patient 4} & 1 & 1.10 & 1.14 & 2.12 & 2.02 & 1.04 & 1.08 & 1.88 & 1.90 \\
\hline & 2 & 1.04 & 1.08 & 2.14 & 2.04 & 0.96 & 1.00 & 1.86 & 1.90 \\
\hline & 3 & 1.04 & 1.06 & 2.16 & 1.90 & 0.98 & 0.98 & 1.86 & 1.76 \\
\hline \multicolumn{2}{|l|}{ Average } & 1.06 & 1.09 & 2.14 & 1.99 & 0.99 & 1.02 & 1.87 & 1.85 \\
\hline \multicolumn{2}{|l|}{$\mathrm{SD}$} & 0.02 & 0.02 & 0.01 & 0.04 & 0.02 & 0.03 & 0.01 & 0.05 \\
\hline \multirow{3}{*}{$\begin{array}{l}\text { Carrier } \\
\text { mother }\end{array}$} & 1 & 2.14 & 2.06 & 2.14 & 2.96 & 2.02 & 1.94 & 1.88 & 2.78 \\
\hline & 2 & 2.08 & 2.04 & 2.08 & 3.00 & 2.00 & 1.96 & 1.92 & 2.88 \\
\hline & 3 & 1.96 & 2.06 & 1.98 & 2.94 & 1.98 & 1.96 & 2.02 & 2.98 \\
\hline \multicolumn{2}{|l|}{ Average } & 2.06 & 2.05 & 2.07 & 2.97 & 2.00 & 1.95 & 1.94 & 2.88 \\
\hline \multicolumn{2}{|l|}{ SD } & 0.05 & 0.01 & 0.05 & 0.02 & 0.01 & 0.01 & 0.04 & 0.06 \\
\hline \multirow{3}{*}{$\begin{array}{l}\text { Male } \\
\text { control }\end{array}$} & 1 & 1.00 & 0.92 & 2.06 & 1.00 & 0.96 & 0.88 & 1.86 & 0.96 \\
\hline & 2 & 1.04 & 1.04 & 2.18 & 1.00 & 0.96 & 0.96 & 1.84 & 0.92 \\
\hline & 3 & 1.00 & 0.94 & 1.94 & 0.96 & 1.02 & 0.98 & 2.08 & 1.00 \\
\hline \multicolumn{2}{|l|}{ Average } & 1.01 & 0.97 & 2.06 & 0.99 & 0.98 & 0.94 & 1.93 & 0.96 \\
\hline \multicolumn{2}{|l|}{ SD } & 0.01 & 0.04 & 0.07 & 0.01 & 0.02 & 0.03 & 0.08 & 0.02 \\
\hline \multirow{3}{*}{$\begin{array}{l}\text { Female } \\
\text { control }\end{array}$} & 1 & 2.06 & 2.04 & 2.02 & 1.96 & 2.04 & 2.02 & 1.98 & 1.94 \\
\hline & 2 & 2.08 & 2.10 & 2.10 & 2.00 & 1.96 & 1.98 & 1.90 & 1.90 \\
\hline & 3 & 2.16 & 2.02 & 2.18 & 2.00 & 1.96 & 1.94 & 1.94 & 1.94 \\
\hline \multicolumn{2}{|l|}{ Average } & 2.10 & 2.05 & 2.10 & 1.99 & 1.99 & 1.98 & 1.94 & 1.93 \\
\hline \multicolumn{2}{|l|}{ SD } & 0.03 & 0.02 & 0.05 & 0.01 & 0.03 & 0.02 & 0.02 & 0.01 \\
\hline
\end{tabular}

SD, standard deviation.

results are graphically summarized in Figure 1. As shown, all four male PMD patients demonstrated two copies of PLP1 and the carrier females demonstrated three copies of PLP1. All triplicates showed similar results (Table 3), and there were no data which showed dispersed values. From these findings, each independent ddPCR result provided precise results.

There are several diagnostic methods to detect CNVs such as FISH, MLPA, chromosomal microarray testing, qPCR, and dPCR (6). At first, the capacity for precise detection of PLPI duplications should be considered. Previously, FISH has been used to detect
PLP1 duplications. In the case of FISH, detected signals are the result of hybridization of labelled DNA probes to corresponding chromosomal regions. Thus, detected signal numbers in a set of karyotype correspond to copy numbers. However, when the targeted regions are small, it is sometimes difficult to detect duplications on the same chromosome, because two signals cannot be separately detected or resolved (3). In this regard, interpretations of FISH results are often subjective.

Reaction conditions for ddPCR may be similar to qPCR. Traditional qPCR, based on relative quantification of target DNA, also can be used to detect 

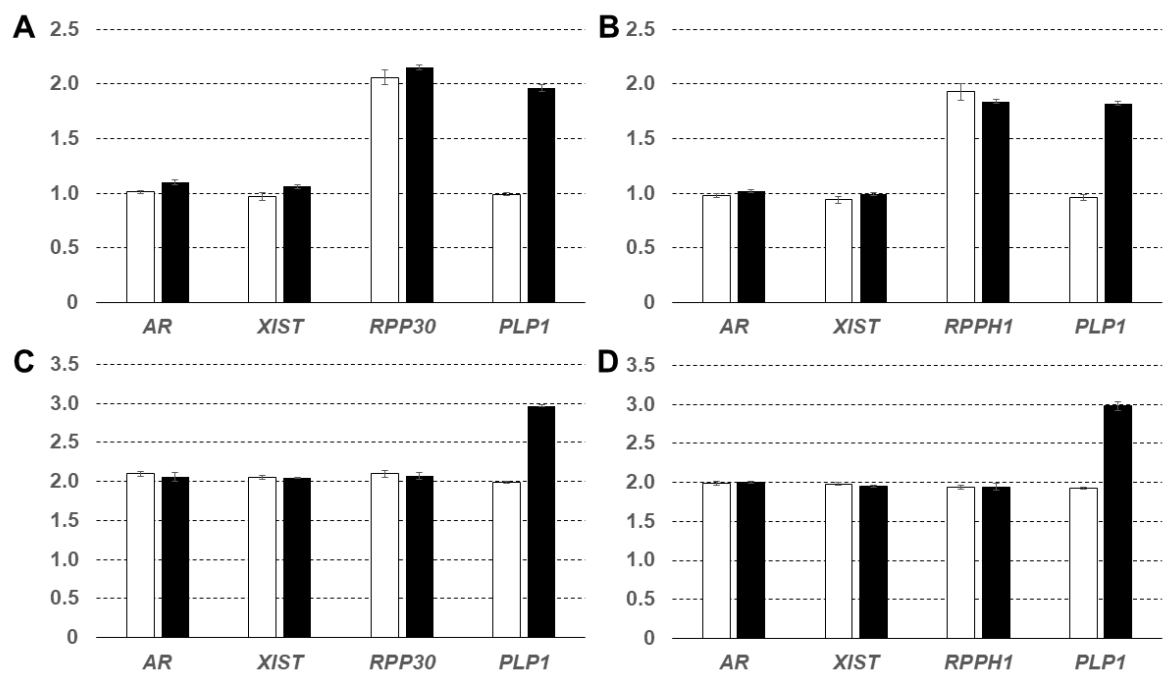

Figure 1. Graphical presentations of droplet digital PCR (ddPCR) results. Average copy number of the triplicates of the targeted genes versus reference genes are shown. Results of male patients (black; $n=4)$ and a normal male control (white; $n=1)$ were compared with RPPH1 (A) and RPP3O (B). Results of a carrier female (white; $n=1$ ) and a normal female control (black; $n$ $=1)$ were also compared with RPPH1 (C) and RPP30 (D). The Y-axis represents copy number estimated by ddPCR. Error bars indicate $\pm 2 \mathrm{SD}$.

genomic copy number. Because qPCR is based on PCR amplification in which DNA is doubled in each amplification cycle, it is usually difficult to detect copy number gains of $1.5 \times$. To compensate for unstable detection, independent triplicate experimental data sets are required for $\mathrm{qPCR}(7,8)$.

In comparison, we should be able to precisely detect small duplications by use of new technologies including MLPA and chromosomal microarray testing. In this study, ddPCR also provided precise results of PLP1 duplications in PMD patients and the carrier mother. For this reason, ddPCR can be used in the same way as same as MLPA and chromosomal microarray testing.

The ddPCR method is a new technology that is based on partitioning template DNA and performing multiple independent PCR amplifications (9). In particular, the ddPCR system was developed to distribute template DNA randomly into emulsions of water-in-oil droplets (10). With data involving positive (containing target DNA) and negative (no target DNA) PCR amplifications, ddPCR provides absolute detection of genomic copy number and precise quantification of target DNA. In this study, results of all triplicates were the same for each sample. These results showed that $\mathrm{ddPCR}$ led to accurate $\mathrm{CNV}$ detection in one experimental $\mathrm{pr}^{\circ} \mathrm{Cess}$, and triplicate experiments are no longer necessary.

Next, we wanted to evaluate assay time required. For FISH analysis, chromosome specimens are needed. For that, at least several days are required. Hybridization also requires time (generally, a number of days). For chromosomal microarray testing, long hybridization times are required (generally overnight). For MLPA, hybridization requires 16 hours. Thus, ddPCR has advantages with regard to required time. As shown, ddPCR requires thermal cycling reactions and droplet read-outs. For these experiments, approximately 6 hours are needed. Compared to other methods, we were able to obtain ddPCR results rapidly.

Furthermore, we wanted to consider required sample amounts. For chromosomal microarray testing, $250 \mathrm{ng}$ is required when we use the Agilent CGH Microarray system (Agilent Technologies, Santa Clara, CA, USA). This is excessive in comparison with MLPA and ddPCR. MLPA generally requires 50-200 ng. ddPCR needs only $20 \mathrm{ng}$. In this regard, ddPCR also has an advantage.

In the case of prenatal diagnosis, carrier mothers will require the result as soon as possible. Regarding sample amounts, we can only extract a small amount of DNA from amniotic fluid (generally, less than 500 ng). Therefore, the necessary time needed and sample amounts required are the critical points for prenatal diagnosis. ddPCR will fulfill these demands with precise results. In conclusion, we showed that ddPCR is a potential diagnostic tool to confirm genomic copy number as a daily clinical application, including prenatal diagnosis.

\section{Acknowledgements}

We would like to thank the patients' families for their cooperation. This research was supported by the Practical Research Project for Rare/Intractable Diseases from the Japan Agency for Medical Research and Development (AMED); a Grant-in-Aid for Scientific Research from the Health Labor Sciences Research Grants from the Ministry of Health, Labor and Welfare, Japan; and the Japan Society for the Promotion of Science (JSPS) KAKENHI grant number 15K09631 (TY). 


\section{References}

1. Inoue K. PLP1-related inherited dysmyelinating disorders: Pelizaeus-Merzbacher disease and spastic paraplegia type 2. Neurogenetics. 2005; 6:1-16.

2. Yamamoto T, Shimojima K. Pelizaeus-Merzbacher disease as a chromosomal disorder. Congenit Anom (Kyoto). 2013; 53:3-8.

3. Shimojima K, Inoue T, Hoshino A, et al. Comprehensive genetic analyses of PLP1 in patients with Pelizaeus-Merzbacher disease applied by array$\mathrm{CGH}$ and fiber-FISH analyses identified new mutations and variable sizes of duplications. Brain Dev. 2010; 32:171-179.

4. Hwang VJ, Maar D, Regan J, Angkustsiri K, Simon TJ, Tassone F. Mapping the deletion endpoints in individuals with 22q11.2 deletion syndrome by droplet digital PCR. BMC Med Genet. 2014; 15:106.

5. Shoda K, Masuda K, Ichikawa D, Arita T, Miyakami Y, Watanabe M, Konishi H, Imoto I, Otsuji E. HER2 amplification detected in the circulating DNA of patients with gastric cancer: A retrospective pilot study.
Gastric Cancer. 2015; 18:698-710.

6. Cantsilieris S, Baird PN, White SJ. Molecular methods for genotyping complex copy number polymorphisms. Genomics. 2013; 101:86-93.

7. Choi YW, Choi JS, Zheng LT, Lim YJ, Yoon HK, Kim YH, Wang YP, Lim Y. Comparative genomic hybridization array analysis and real time PCR reveals genomic alterations in squamous cell carcinomas of the lung. Lung Cancer. 2007; 55:43-51.

8. Liu C, Cao R, Xu Y, Li T, Li F, Chen S, Xu R, Sun K. Rare copy number variants analysis identifies novel candidate genes in heterotaxy syndrome patients with congenital heart defects. Genome Med. 2018; 10:40.

9. Quan PL, Sauzade M, Brouzes E. dPCR: A technology review. Sensors (Basel). 2018; 18. pii: E1271.

10. Hindson BJ, Ness KD, Masquelier DA, et al. Highthroughput droplet digital PCR system for absolute quantitation of DNA copy number. Anal Chem. 2011; 83:8604-8610.

(Received May 27, 2019; Revised August 5, 2019; Accepted August 10, 2019) 